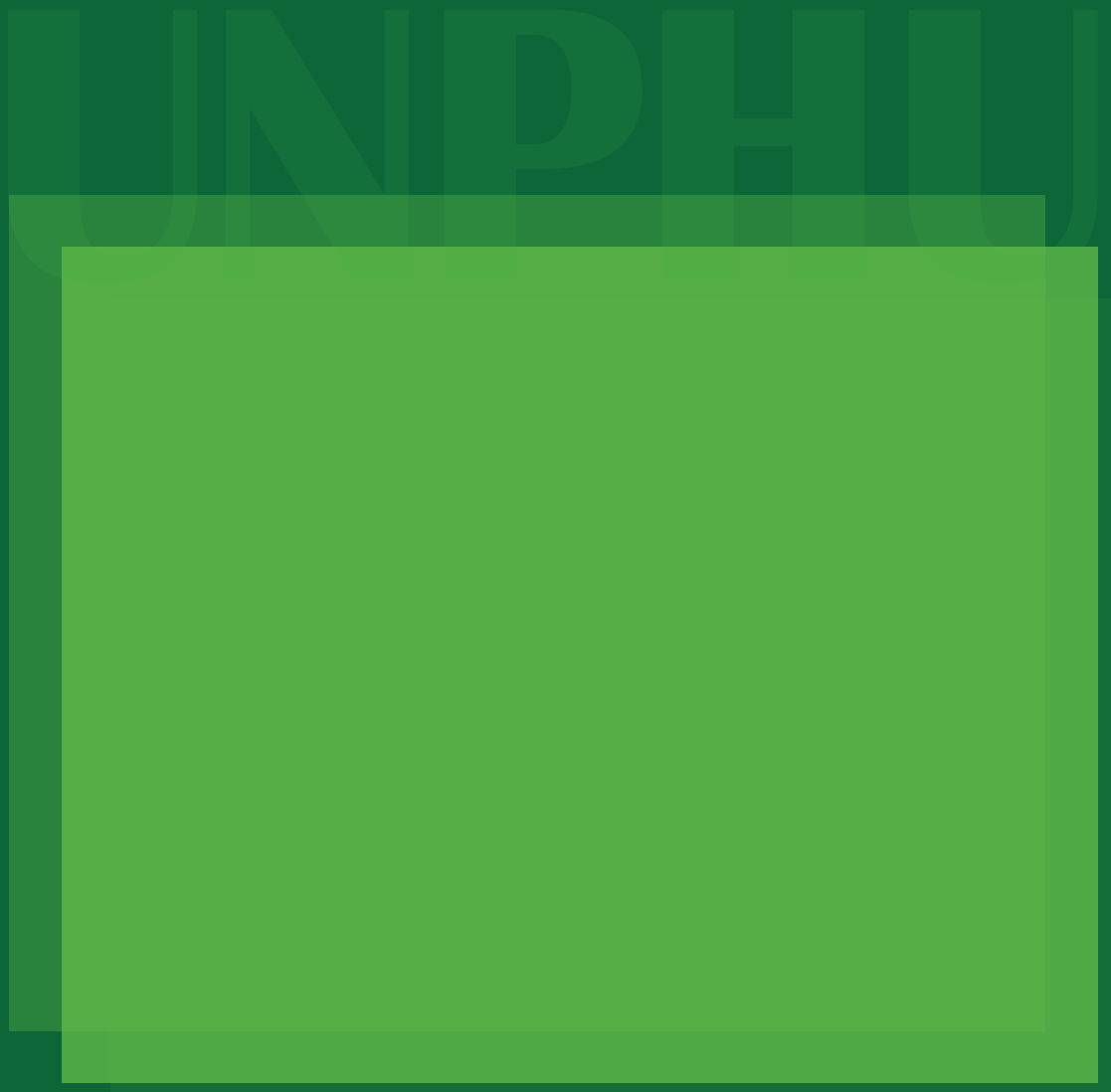




\title{
El medio ambiente sostenible de la mano del desarrollo económico
}

\section{The sustainable environment hand in hand with the economic development}

\author{
Francis Meriño \\ Profesora de Hotelería y Turismo, UNPHU \\ Santo Domingo, República Dominicana \\ fm5549@unphu.edu.do
}

Fecha de recepción: 8 de enero de 2018.

Fecha de aceptación: 12 de abril de 2018

Favor de citar este artículo de la siguiente forma:

Meriño, F. (2018) El medio ambiente sostenible de la mano del desarrollo económico.

Revista AULA, Vol 62. Número 2, enero junio 2018. Santo Domingo: Amigo del Hogar.

\section{RESUMEN}

El desarrollo económico de una nación está claramente determinado por un manejo efectivo de sus recursos para la producción, desarrollo y crecimiento y por lo tanto asociamos que el progreso de uno afecta el avance del otro. Las investigaciones científicas nos demuestran además, que el calentamiento global ha sido causado por el afán por las riquezas de las industrias, por lo que es perentorio que los gobiernos incluyan políticas de acción y preservación, claras y universales para cualquier nación, sin dejar de involucrarnos personalmente en nuestro accionar en materia de competitividad y consumismo. La República Dominicana con un desarrollo económico en continuo crecimiento, ha establecido dentro de sus políticas de desarrollo acciones que fomenten el consumo sostenible y que promuevan la conservación de nuestros recursos naturales asegurando de esta manera la continuidad de esta evolución y sobre todo estableciendo los lineamientos para que las nuevas industrias y la generación entrante, tengan un soporte estructural sostenido en todos los renglones que afectan el buen desarrollo de esta isla. Al menos en teoría, la Republica Dominicana está a la vanguardia de las tendencias globales con políticas públicas enfocadas al desarrollo sostenible. Para mencionar algunos casos recientes la constitución de 2010 incluye e incorpora premisas importantes para el tema ambiental, la creación del Consejo Nacional para el Cambio Climático y el Mecanismo de Desarrollo Limpio, la estrategia nacional de desarrollo 2030, la Ley de Bioseguridad 219-15 y la Ley de Biodiversidad 333-15, y algunos proyectos de Ley como Pagos por Servicios Ambientales y la Ley de Agua que actualmente cursan los estamentos legislativos dominicanos. Sin embargo, es pertinente que este desarrollo sostenible se alimente con una realidad que debemos incorporar, una perspectiva a largo plazo que apuntale al manejo de estos recursos y que enfatiza en la solidaridad hacia las actuales y futuras generaciones defendiendo la equidad intergeneracional.

Palabras clave: cuidado ambiental, desarrollo sostenible, economía, equidad intergeneracional, recursos naturales, sustentabilidad. 


\begin{abstract}
The economic development of a nation is clearly determined by an effective management of its resources for production, development and growth and therefore we associate that one's progress affects the progress of the other. Scientific research also shows that global warming has been caused by the enthusiasm for the riches of industries so it is peremptory that governments include policies of action and preservation, clear and universal for any nation, without failing to personally engage in our actions in terms of competitiveness and consumerism. It's a commitment from everyone. The Dominican Republic with a growing economic development, has established within its development policies actions that promote sustainable consumption and promote the conservation of our natural resources thus ensuring the Continuity of this evolution and especially establishing the guidelines for the new industries and the incoming generation, have a sustained structural support in all lines that affect the good development of this I of this island. At least in theory, the Dominican Republic is at the global trends forefront with public policies focused on sustainable development. To mention some recent cases, the 2010 Constitution includes and incorporates important premises for the environmental issue, the creation of the National Council for Climate Change and the clean development Mechanism, the national Development Strategy 2030, the Biosecurity law 219-15 and the Biodiversity Law 333-15 and some projects Bills such as payments for environmental services and the water law currently being pursued by the Dominican legislative entities. However, it is pertinent that this sustainable development is fed with a reality that we must incorporate, a long-term perspective that points to the management of these resources and that emphasizes in the solidarity towards the present and future generations defending the Intergenerational equity.
\end{abstract}

\title{
Palabras clave: Sustainable development, economy, sustainability, environmental care, natural resources, intergenerational equity
}

\section{El medio ambiente sostenible de la mano del desarrollo económico}

En la antigüedad, los países eran categorizados dependiendo de su desarrollo hegemónico y la posesión territorial. Hoy en día, se consideran potencia mundial a aquellas naciones que poseen un marcado desarrollo económico.

Sin entrar en detalles de las diversas acepciones filosóficas de lo que significa desarrollo, tomaremos como punto de partida que todo desarrollo y crecimiento económico implica la producción masiva de bienes y servicios (Barreiro, Azcona, \& Morcillo, 1999). Esto genera tres procesos consecutivos y dependientes: crecimiento, producción y consumo.

Los recursos para la producción, desarrollo y crecimiento salen de nuestra madre naturale- za, por lo que se asocia que el desarrollo de uno afecta el desarrollo del otro.

Las acciones del hombre en busca de su bienestar transforman las condiciones del medio ambiente, y no siempre estos cambios son favorables para sostener el bienestar que buscamos ahora, y que esperamos encontrar en el futuro.

Desde hace varias décadas las actividades humanas tienen que ver con fomentar el desarrollo económico, y eso tiene un efecto concreto en el medio ambiente: lo transforma. Aunque no se debe generalizar, lo cierto es que el paso del tiempo ha demostrado que el modelo económico vigente se caracteriza por una explo- 
tación excesiva de los recursos naturales y por generar altos niveles de contaminación al medio ambiente, por lo tanto el hombre transforma el medio de una manera negativa.

Se destruyen montañas para extraer minerales, se talan bosques para conseguir madera, se sobreexplotan los acuíferos, se extinguen especies animales, se llena el aire de gases tóxicos. (Pérez Ventura, 2014).

Las publicaciones relacionadas al medio ambiente nos hablan de un cambio gradual de las temperaturas de la atmósfera y los océanos, la tala indiscriminada, los incendios forestales, y la sequía de ríos, lagos y lagunas.

Las investigaciones científicas nos demuestran que el calentamiento global ha sido causado por el afán por las riquezas, reflejado en la explotación de los yacimientos mineros, la desforestación y el auge de las industrias.

El ecoturismo es el segmento con mayor crecimiento en el mundo y con mayor potencial para integrar las dimensiones de la sostenibilidad. Es una actividad que, en la mejor de sus acepciones, produce un mínimo impacto en el ambiente, y relaciona aspectos de aprendizaje con la conservación, la comprensión y el aprecio por el entorno y por las culturas que se visitan.

La respuesta en nuestro país a este nuevo paradigma de hacer negocios, se aprecia en el incremento de ofertas turísticas que permiten esta interacción hombre-naturaleza: Cabarete Maravilla Eco Logde, Villa Pajón, Valle Nuevo, Constanza, Paraíso Eco Lodge, Punta Rucia, Samana Eco Lodge en Las Terrenas; para mencionar algunos de los puntos de nuestra isla, que ya han destacado en la práctica del manejo de los recursos naturales de manera sustentable.
Las ofertas de turismo han permitido que otras regiones de nuestra isla crezcan con este modelo inequívoco y como parte del principio de sustentabilidad de las actividades humanas, en las cuales la economía, el bienestar y el ambiente forman un todo.

La creación del Acuerdo de París es un claro ejemplo que reconoce esta realidad, y la firma de sus miembros representa un compromiso de velar por medidas económicas que no tengan ningún impacto en el medio ambiente, o en su defecto que reduzcan tal consecuencia. Otros sostienen que la carga medioambiental está mal distribuida y países como Estados Unidos, a través de su presidente Donald Trump, ha dicho que «El concepto de calentamiento global fue creado por los chinos, para volver a la industria manufacturera estadounidense poco competitiva» (Nov. 6, 2012). Esto significa que ha preferido el crecimiento de sus empresas como prioridad a su contribución global con el medio ambiente. Cuando el líder de una potencia económica como los EUA, se expresa de esta manera, la tendencia es dejar de "proteger" y enfocarnos en "producir", porque somos minoría y no podemos pretender ganar esta batalla en la mente de todos los involucrados.

La principal dificultad al medir el impacto económico del turismo es que el impacto total es la suma de los efectos directos, indirectos e inducidos dentro de una región y no hay datos que puedan reflejar estos aspectos.

El turismo no es una industria en el sentido tradicional, sino más bien una actividad que se lleva a cabo por una serie de sectores de la industria (incluido el alojamiento, la restauración, el transporte, el entretenimiento y el comercio al por menor), por lo que medir el impacto económico del turismo es una cuestión compleja. 
En su versión 2016-2017, el Índice Global de Competitividad emitido por el Foro Económico Mundial, evalúa 138 economías. La República Dominicana ocupa la posición 92 a nivel mundial y la 13 a nivel regional, destacándose entre las economías de más rápido crecimiento en el índice, y exhibiendo una puntuación de 3.94 en un rango del 1-7.

Según la metodología del IGC, República Dominicana es considerada una economía conducida por la eficiencia, es decir, los pilares que tienen mayor peso en su ponderación son aquellos que miden el desarrollo de sus procesos productivos.

Al revisar todos los indicadores del índice, podemos concluir que el ligero aumento del desempeño general del país, se debe en gran medida a los indicadores macroeconómicos incluidos en el Pilar de Ambiente Macroeconómico, el cual tiene un peso de alrededor del $10 \%$ del total del índice.

También en el reporte del índice se resalta al país como un ejemplo de la asociación de los resultados del Índice con las Políticas Públicas a nivel nacional.

Aunque se establece con estos informes aspectos positivos de crecimiento, el cuidado del Medio Ambiente, necesita estar regulado y no es hasta el año 2000 con la promulgación de la Ley 64, y posteriormente reconocida en la Constitución, que se inicia esta normalización.

La Ley 64-00 tiene como finalidad principal conservar, proteger, mejorar y restaurar el medio ambiente y los recursos naturales con miras de asegurar su uso sostenible, declarándolos como interés nacional y como una responsabilidad del Estado, de la sociedad y de cada habitante. Uno de sus aspectos más importantes es la enumeración de los delitos ecológicos o medioambientales que lo posicionan como un bien jurídico penal; además de la creación de una Procuraduría especial para el medio ambiente como órgano encargado de la persecución de los delitos ambientales.

El deber del Estado en cuanto a la garantía de protección del medio ambiente tiene rango constitucional; sin embargo, no es hasta el año 2010 cuando el medio ambiente encuentra una protección constitucional. La norma suprema dominicana instituye claramente que el Estado debe garantizar de modo individual y colectivo el uso y goce sostenible de los recursos naturales.

La razón de ser del derecho penal como medio de control social y jurídico es evitar determinados comportamientos sociales que se consideran incorrectos o indeseables, recurriendo a la amenaza de imposición de diferentes sanciones en caso de que dichos actos se cometan.

La puesta en marcha de la acción penal ambiental inicia con la creación, como mencionamos anteriormente, de una Procuraduría especializada en la materia. De esta manera, la denominada Procuraduría para la Defensa del Medio Ambiente y los Recursos Naturales representa y ejerce la defensa del interés del Estado y la sociedad en los aspectos relacionados al medio ambiente.

El procedimiento para el inicio de la investigación y sometimiento por delitos ambientales está estipulado en el Código Procesal Penal que establece aspectos fundamentales y supletoriamente por el Derecho común, de donde podemos ver una armonía evidente entre las disposiciones de este código y la Ley. Tenemos entonces un índice de Competitividad favorecedor, que nos presenta en franco crecimiento, pero constitucionalmente tenemos solo 8 años siendo regulados. 
Como preámbulo para entender la puesta en escena del marco regulatorio, y nutridos de una información que nos ayudará a realizar un examen exhaustivo de los avances que han realizado en esta materia, revisamos el Plan Estratégico anual 2017, del Portal del Ministerio de Medio Ambiente y Recursos Naturales de la República Dominicana.

Encontramos que los avances más significativos alcanzados a la fecha están indicados dentro del capítulo Visión de la Nación de Largo Plazo 2030, cuarto eje, que procura una Sociedad de Producción y Consumo Ambientalmente Sostenible que Adapta al Cambio Climático.- "Una sociedad con cultura de producción y consumo sostenible, que gestiona con equidad y eficacia los riesgos y la protección del medio ambiente y los recursos naturales y promueve una adecuada adaptación al cambio" (Ver anexos).

En papel, están bien definidos, la continuidad y seguimiento a estos objetivos nos desafía con grandes beneficios, así como, grandes retos por alcanzar, si los directivos mantienen realmente, esta línea de acción y si la conciencia de nuestro gobierno, invierte en mayores montos para poder garantizar la sostenibilidad de estos proyectos y sus obvios beneficios para nuestra nación.

La idea es adoptar estilos de vida y pautas de desarrollo que respeten los límites de la naturaleza y se desenvuelvan dentro de estos límites. Este nuevo enfoque deberá compensar dos requisitos fundamentales: uno es lograr un compromiso profundo y generalizado con una nueva ética, la ética de vivir de manera sostenible, y materializar sus principios en la práctica. El otro consiste en integrar la conservación y el desarrollo: la conservación que mantiene nuestras acciones dentro de la capacidad de carga de la Tierra, y el desarrollo que permite a todo el mundo disfrutar de una vida prolongada, saludable y satisfactoria.
Es de considerar la importancia de evaluarnos sistemáticamente como se ha venido haciendo desde septiembre del año 2000, durante la asamblea general de las Naciones Unidas, con la firma de la Declaración de los Objetivos del Milenio donde participaron 189 países signatarios, entre cuyos representantes estaban 147 Jefes de Estado y Gobierno, incluyendo al entonces presidente de República Dominicana, Hipólito Mejía. Como eje central de esta declaración, los países se comprometieron a realizar todos los esfuerzos necesarios para alcanzar unos Objetivos de Desarrollo del Milenio, buscando hacer del mundo un lugar mejor.

Los años donde existen informes de avance para el país corresponden al 2004, 2008 y 2010. En estos se presentan la evaluación más completa del progreso alcanzado hasta la fecha y se basa en información procedente tanto de instituciones gubernamentales, como de organizaciones internacionales.

Con este monitoreo se han podido identificar oportunidades de mejora y brechas por atender, que asumo se tomaron en cuenta para la preparación del Plan Estratégico 2017, preparado por el Ministerio de Medio ambientes, donde se incluyen y comparten aprendizajes y experiencias con vecinos y aliados, se crean mejores redes de conocimiento, incrementamos la legitimidad y credibilidad de los procesos de desarrollo, y ayudan, con los ajustes internos necesarios, a corregir el curso y avanzar en el trayecto de la mejor manera posible.

La República Dominicana firmó los acuerdos de los Objetivos de Desarrollo Sostenible (ODS), los cuales están basados en los Objetivos de Desarrollo del Milenio e incluyen temas como el cambio climático y el consumo sostenible comprometiéndose a integrar dentro de sus políticas de desarrollo acciones que fomen- 
ten el consumo sostenible y que promuevan la conservación de nuestros recursos naturales.

Durante las últimas décadas, los gobiernos dominicanos han concentrado sus esfuerzos en el desarrollo y crecimiento económico y el medio ambiente, bajo el entendido de que estas áreas son interdependientes.

Temas tan diversos como el manejo de sustancias peligrosas, residuos, recursos naturales, suelo, energía y áreas protegidas ya no son exclusividad de los medioambientalistas al punto de que nuestra nación cuenta con un marco legal regulatorio, así como de auditores capacitados en las áreas de seguridad, higiene, y medio ambiente.

Esta realidad implica que se ha asimilado que una nación no puede tener un desarrollo y crecimiento económico sostenido si no preserva y desarrolla el medio ambiente de manera que aseguremos la continuidad de la vida en el planeta.

Con medidas económicas que garanticen que los recursos naturales serán utilizados a un ritmo adecuado, con la preservación de recursos no renovables, manejo de desechos sin impacto medioambiental y políticas de uso y explotación de nuestros bienes, República Dominicana puede lograr un buen desarrollo económico que les permita a sus habitantes sostenerse y llevar una vida sana.

Revisando también las recién presentadas estadísticas publicadas por el Banco Mundial en enero de 2018, para introducirnos en la panorámica global, nos presenta un escenario donde se advierte que se duplicará para América Latina la proyección realizada, ya que el crecimiento de la economía global está siendo más robusto de lo esperado.
La expansión fue del 3\% el pasado año y el Banco Mundial proyecta que se mantendrá en el 3,1\% el que acaba de arrancar. Es un repunte que califica de "notable" cuando se compara con el 2,4\% de 2016. La tendencia se acelera para América Latina. Los países de la región se alejan de la recesión y duplicarán el ritmo de crecimiento al 2\% en 2018, frente al $0,9 \%$ en 2017.

La nueva estimación para la economía global se mejora en dos décimas frente a lo adelantado en junio para 2018. La lectura de 2017 es tres décimas más alta. Este mejor comportamiento se explica por un impulso de la inversión en los países avanzados y a una aceleración de la actividad en los emergentes, que crecerá al 4,5\% este año.

La recuperación, como señala el Banco Mundial, está mejor repartida. "El crecimiento mejoró en más de la mitad de las economías en 2017”, señala el informe. Y aunque las curvas muestran que la tendencia seguirá en 2018, anticipa que se moderará de forma gradual a lo largo de 2019 porque la inversión en los países avanzados se relajará, y por una desaceleración de la demanda en China.

La proyección para la región es muy parecida a la que se anticipó hace seis meses, con ajustes de una décima al alza para 2017 y a la baja para 2018. El consumo privado es el principal sustento a la actividad económica. El crecimiento habría sido mayor si la inversión no hubiera sido tan modesta.

En el caso concreto de América Latina, señala que el impulso externo al crecimiento será menor porque la expansión en Estados Unidos y en China tenderá a moderases en 2019 y 2020. "La región necesitará depender más de los recursos internos que en el pasado", anticipa el Banco Mundial. También indica que el poten- 
cial seguirá siendo inferior a la media histórica previa a la crisis, por la baja productividad.

El incremento del proteccionismo, junto a las tensiones geopolíticas, también jugará en contra a corto plazo. "La escalada de las restricciones puede hacer descarrilar la recuperación y deshacer todo lo que se ganó con los pasados esfuerzos liberalizadores", alerta el informe.

También se cita la disrupción que pueden crear los desastres naturales, en especial en los países donde el margen fiscal es más reducido.

En la República Dominicana, a través de la más reciente Evaluación de Resultados del Programa de las Naciones Unidas para el Desarrollo (PNUD), realizada con el concurso de varios actores de la cooperación al desarrollo, se identificaron importantes hallazgos y lecciones que fueron incorporadas en el diseño del nuevo Documento de Programa País del PNUD para la República Dominicana.

Un desarrollo sustentable, como se ha definido a este cambio, implica un nuevo modelo que sea socialmente justo y ecológicamente equilibrado. Este cambio deberá inscribirse en transformaciones relativas a la sociedad (medio ambiente humano) y que se relacionen con el manejo y utilización de los recursos naturales (medio ambiente natural).

"La región necesitará depender más de los recursos internos que en el pasado" cito de nuevo al Banco Mundial, para establecer un punto relevante que no se debe descuidar. Pero el futuro es prometedor, no estamos inactivos, reconocemos el valor económico de la sustentabilidad y el cuidado de nuestros recursos naturales. Lo que nos preocupa ahora es la educación, la concienciación de las generaciones actuales y las venideras, y la promesa de mantener nuestra bella isla, competitiva, contrastante en sus escenarios naturales, fértil, exuberante, y que sobre todo, razonemos que no es un filón de oro, sin fin.

Existe la posibilidad, muy cercana, de que nos quedemos sin respuestas, que no podamos accionar a tiempo para proveernos de medios rentables a lo largo de nuestras vidas, y está en cada uno nosotros, de manera individual y después colectiva, poner nuestro grano de arena preventivo

Se hace necesario que todos los habitantes de la Tierra nos cuestionemos sobre los procesos que generan producción y consumo, de manera que tal reflexión nos ayude a encontrar maneras propicias de enfrentar los cambios que debemos realizar para seguir abasteciendo nuestras necesidades sin dañar nuestro entorno.

Los incendios forestales, la tumba y quema para el conuquismo, la eliminación de manglares, el rellenado de humedales, la contaminación de ríos y otras acciones del ser humano que ponen el peligro diferentes especies, es noticia cada cierto tiempo aún. ¿Está realmente el Estado trabajando con la gente para que tome conciencia de los daños que provoca y realice acciones para mitigarlos?

Debemos mirar cuidadosamente nuestro accionar en materia de competitividad y consumismo. Se hace perentorio que las organizaciones eliminen de sus prácticas aquellas medidas que, en busca de minimizar costos, explotan indiscriminada e intensivamente los recursos naturales.

La idea es adoptar estilos de vida y pautas de desarrollo que respeten los límites de la naturaleza y se desenvuelvan dentro de estos límites. Este nuevo enfoque deberá compensar dos requisitos fundamentales: uno es lograr un compromiso profundo y generalizado con una 
nueva ética, la ética de vivir de manera sostenible, y materializar sus principios en la práctica. El otro consiste en integrar la conservación y el desarrollo: la conservación que mantiene nuestras acciones dentro de la capacidad de carga de la Tierra, y el desarrollo que permite a todo el mundo disfrutar de una vida prolongada, saludable y satisfactoria.

El Estado debe garantizar y ratificar en cada periodo electoral este compromiso, y asignar mayores recursos económicos que los actuales. La República Dominicana tiene todos los ingredientes para convertirse en un destino sólido y el aporte de esta continuidad, tendrá como resultados, informes con objetivos logrados y todos alineados, así como un horizonte claro e indicativo de nuestro desarrollo económico para beneficio de la próxima generación.

\section{Referencias}

Alvigini, Antonella. AEI, Regimen Penal ambiental de la República Dominicana. Tomado de: http://www.encuentrojuridico.com/2013/12/el-regimen-penal-ambiental-de-la.html

Aravena, C., Escobar, L., y Hofman, A. (2015). Serie 164 Fuentes del crecimiento económico y la productividad en América Latina y el Caribe, 1990-2013. Tomado de: http://repositorio.cepal.org/bitstream/handle/11362/38276/S1500525_ es.pdf? sequence $=1 \&$ isAllowed $=\mathrm{y}$

Barreiro P, F., Azcona, J. L., y Morcillo, F. M. (1999). Macroeconomía intermedia. McGraw-Hill Interamericana de España SAU, Madrid, España.

Depósito de Documentos de la Organización de las Naciones unidas para la Alimentación y la Agricultura (FAO). Tomado de http://www.fao.org/docrep/x5600s/ x 5600s05.htm

Informes Índice Global de la Competitividad Republica Dominicana: http://www.com petitividad.org.do/wp-content/ uploads/2016/09/Desempe \%C3\%B1o-de-Rep \%C3\%BAblica-Dominicana-en-el-Indice-Global-de-Competitividad.pdf

Instituto Latinoamericano de Planificación Económica y Social (ILPES) (1968). Cinco modelos de crecimiento económico. Cuadernos de ILPES, 1(3), 1-108.

Ley General sobre Medio Ambiente y Recursos Naturales. Disponible en: https://observatoriop10.cepal.org/es/instrumentos/ ley-general-medio-ambiente-recursos-naturales-ley-no-64

Pérez, V. J. (2014). Crecimiento económico y conservación del medio ambiente. $E l$ Orden Mundial en el Siglo XXI. Tomado de https://elordenmundial.com/category/ economia/

Periódico digital El PAIS: https://elpais.com/ economia/2018/01/09/actuali $\mathrm{dad} / 1515500241$ 937177.html

Portal Web de la Secretaria de Medio ambiente: http://ambiente.gob.do/

Programa de las Naciones Unidas para el Desarrollo (PNUD) (2014). República Dominicana en breve. Tomado de http:// www.do.undp.org/content/dominican republic/es/home/countryinfo.html\#Intro

Sistema de Información de los Objetivos de Desarrollo del Milenio (ODM), sitio oficial de la República Dominicana para informar sobre los avances alcanzados en la materia. http://odm.gob.do/ 
Anexo

Síntesis de la Ley No. 01-12 de Estrategia Nacional de Desarrollo 2030:

Visión de la nación a largo plazo: ejes estratégicos, políticas transversales, objetivos generales, específicos y líneas de acción, indicadores y metas, y reformas asociadas al cuarto eje.

\section{Visión de la nación de largo plazo (2030)}

"República Dominicana es un país próspero, donde las personas viven dignamente, apegadas a valores éticos y en el marco de una democracia participativa que garantiza el Estado social y democrático de derecho y promueve la equidad, la igualdad de oportunidades, la justicia social que gestiona y aprovecha sus recursos para desarrollarse de forma innovadora, sostenible y territorialmente equilibrada e integrada y se inserta competitivamente en la economía global".

\section{Ejes estratégicos}

Primer Eje, que procura un Estado Social Democrático de Derecho.- "Un Estado social y democrático de derecho, con instituciones que actúan con ética, transparencia y eficacia al servicio de una sociedad responsable y participativa, que garantiza la seguridad y promueve la equidad, la gobernabilidad, la convivencia pacífica y el desarrollo nacional y local”.

Segundo Eje, que procura una Sociedad con Igualdad de Derechos y Oportunidades.- "Una sociedad con igualdad de derechos y oportunidades, en la que toda la población tiene garantizada educación, salud, vivienda digna y servicios básicos de calidad, y que promueve la reducción progresiva de la pobreza y la desigualdad social y territorial".
Tercer Eje, que procura una Economía Sostenible, Integradora y Competitiva.- "Una economía territorial y sectorialmente integrada, innovadora, diversificada, plural, orientada a la calidad y ambientalmente sostenible, que crea y desconcentra la riqueza, genera crecimiento alto y sostenido con equidad y empleo digno, y que aprovecha y potencia las oportunidades del mercado local y se inserta de forma competitiva en la economía global”.

Cuarto Eje, que procura una Sociedad de Producción y Consumo Ambientalmente Sostenible que Adapta al Cambio Climático.- "Una sociedad con cultura de producción y consumo sostenible, que gestiona con equidad y eficacia los riesgos y la protección del medio ambiente y los recursos naturales y promueve una adecuada adaptación al cambio climático".

\section{Políticas transversales}

Derechos Humanos.- Todos los planes, programas, proyectos y políticas públicas deberán incorporar el enfoque de derechos humanos en sus respectivos ámbitos de actuación, a fin de identificar situaciones de vulneración de derechos, de discriminación o exclusión de grupos vulnerables de la población y adoptar acciones que contribuyan a la equidad y cohesión social

Enfoque de Género.- Todos los planes, programas, proyectos y políticas públicas deberán incorporar el enfoque de género en sus respectivos ámbitos de actuación, a fin de identificar situaciones de discriminación entre hombres y mujeres y adoptar acciones para garantizar la igualdad y la equidad de género.

Sostenibilidad Ambiental.- Todos los planes, programas, proyectos y políticas públicas deberán incorporar criterios de sostenibilidad ambiental y adecuada gestión integral de riesgos. 
Cohesión Territorial.- En el diseño y gestión de las políticas públicas deberá incorporarse la dimensión de la cohesión territorial y asegurar la necesaria coordinación y articulación entre dichas políticas, a fin de promover un desarrollo territorial más equilibrado mediante la dotación de infraestructura, servicios y capacidades necesarias para impulsar el desarrollo de las regiones y los municipios menos prósperos y promociona estrategias regionales de desarrollo y competitividad que aprovechen la diversidad regional, con el concurso de los gobiernos locales y actores sociales, económicos y políticos de cada región.

Participación Social.- Deberá promoverse la participación social en la formulación, ejecución, auditoría y evaluación de las políticas públicas, mediante la creación de espacios y mecanismos institucionales que faciliten la corresponsabilidad ciudadana, la equidad de género, el acceso a la información, la transparencia, la rendición de cuentas, la veeduría social y la fluidez en las relaciones Estado-sociedad.

Uso de las Tecnologías de la Información y la Comunicación.- En el diseño y ejecución de los programas, proyectos y actividades en que se concretan las políticas públicas, deberá promoverse el uso de las tecnologías de la información y comunicación como instrumento para mejorar la gestión pública y fomentar una cultura de transparencia y acceso a la información, mediante la eficientización de los procesos de provisión de servicios públicos y la facilitación del acceso a los mismos.

Responsabilidad Institucional.- La reglamentación de esta ley establecerá los organismos públicos responsables de velar por el cumplimiento de las disposiciones de este capítulo y definir los requisitos mínimos para la aplicación de las políticas transversales. 155
Artículo 27. Objetivos Específicos y Líneas de Acción. Los Objetivos Generales, Específicos y Líneas de Acción correspondientes a cada uno de los Objetivos Generales del Cuarto Eje Estratégico son: EJE 4

Una sociedad con cultura de producción y consumo sostenibles, que gestiona con equidad y eficacia los riesgos y la protección del medio ambiente y los recursos naturales y promueve una adecuada adaptación al cambio climático.

\section{Objetivos específicos}

Objetivo General 4.1

Manejo sostenible del medio ambiente

4.1.1 Proteger y usar de forma sostenible los bienes y servicios de los ecosistemas, la biodiversidad y el patrimonio natural de la nación, incluidos los recursos marinos.

\section{Líneas de acción}

4.1.1.1 Fortalecer, a nivel nacional, regional y local, la institucionalidad, el marco regulatorio y los mecanismos de penalización para garantizar la protección del medio ambiente conforme a los principios del desarrollo sostenible.

4.1.1.2 Fortalecer la participación de los gobiernos locales en la gestión del medio ambiente y los recursos naturales y promover su implementación, en el ámbito geográfico del Plan de Ordenamiento Territorial.

4.1.1.3 Promover un sistema de Manejo Integral de Zonas Costeras, asignando prioridad a las áreas no protegidas.

4.1.1.4 Establecer prioridades de inversión pública en las Grandes Regiones Estratégicas de Planificación del Desarrollo, en función de la sostenibilidad ambiental de cada una de ellas. 
4.1.1.5 Fortalecer las capacidades profesionales y recursos tecnológicos para la gestión ambiental y el desarrollo sostenible a partir de las potencialidades que presentan las Grandes Regiones Estratégicas de Planificación.

4.1.1.6 Desarrollar sistemas de monitoreo, evaluación y valoración del estado del medio ambiente y los recursos naturales a nivel nacional, regional y local, a partir de la consolidación de un Sistema de Información Ambiental que incluya la valoración de los recursos naturales en las cuentas nacionales.

\subsubsection{Realizar investigaciones y crear} sistemas de información y análisis sistemáticos acerca del impacto de la degradación del medioambiente en las condiciones de vida de la población, en particular sobre las mujeres y los grupos vulnerables.
4.1.1.8 Restaurar y preservar los servicios prestados por los ecosistemas, con énfasis en las cuencas de los ríos, y diseñar e instrumentar mecanismos para el pago de servicios ambientales a las comunidades y unidades productivas que los protejan.

4.1.1.9 Gestionar los recursos forestales de forma sostenible y promover la reforestación de los territorios con vocación boscosa con especies endémicas y nativas.

4.1.1.10 Incentivar el uso sostenible de los recursos naturales, mediante la aplicación de instrumentos económicos y de mercado, incluidos los Mecanismos de Desarrollo Limpio.

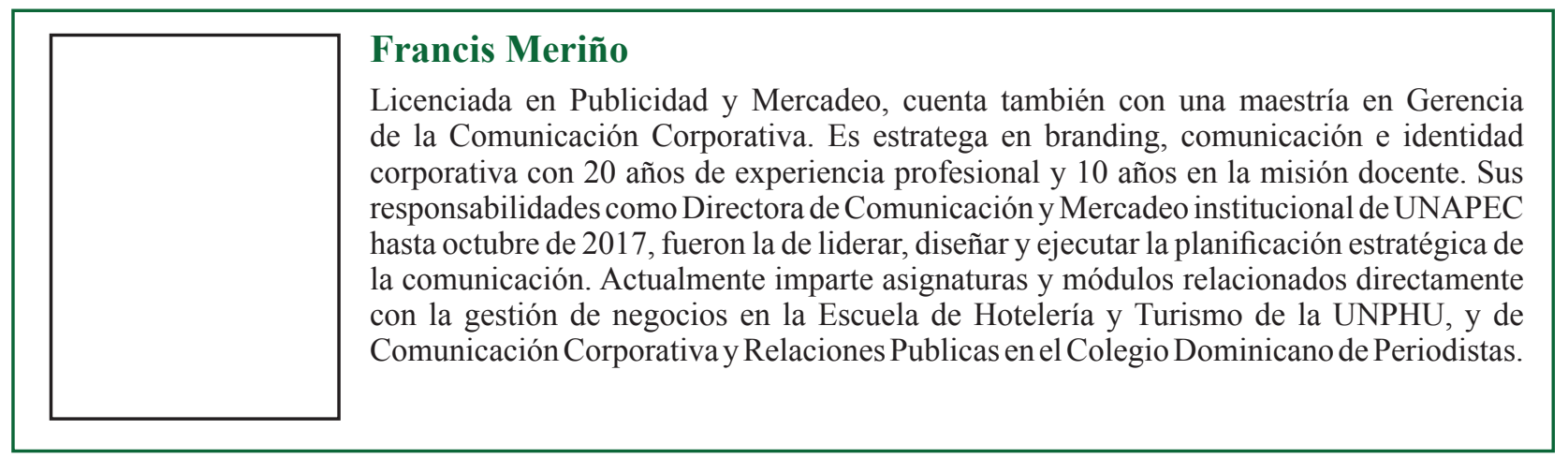

